\title{
Identification of Backbone Curves and Nonlinear Frequency Responses using Control-based Continuation and Local Gaussian Process Regression
}

\author{
L. Renson \\ Department of Mechanical Engineering, Imperial College London, London, UK.
}

\begin{abstract}
Control-based continuation (CBC) is a general and systematic method to probe the dynamics of nonlinear experiments. In this paper, $\mathrm{CBC}$ is combined with a novel continuation algorithm that is robust to experimental noise and enables the tracking of important nonlinear dynamic features such as backbone and nonlinear frequency response curves. The method uses Gaussian process regression to create a local model of the response surface on which standard numerical continuation algorithms can be applied. The local model evolves as continuation explores the experimental parameter space, exploiting previously captured data to actively select the next data points to collect such that they maximise the potential information gain about the feature of interest. The method is demonstrated experimentally on a nonlinear structure featuring harmonically-coupled modes. The regression model is also exploited to estimate the uncertainty of the identified features.
\end{abstract}

Keywords: nonlinear vibrations, experimental testing, control-based continuation, backbone curves, nonlinear frequency response, Gaussian process regression, uncertainty quantification.

\section{INTRODUCTION}

Without the need for a mathematical model, control-based continuation (CBC) is a means to apply the principles of numerical continuation directly to a physical system and experimentally identify key dynamic features such as nonlinear frequency response curves $(\mathrm{NLFR})^{[1]}$, backbone curves $^{[2]}$ and isola ${ }^{[3]}$. Initially proposed by Sieber and Krauskopf ${ }^{[4]}$, CBC has been experimentally demonstrated on a range of mechanical systems, including a parametrically-excited pendulum ${ }^{[5]}$, an impact oscillator ${ }^{[6]}$ and a cantilever beam with a nonlinear mechanism at its free tip ${ }^{[3]}$.

The fundamental idea of CBC is to use feedback control to stabilise the dynamics of the experiment whilst making the control system non-invasive such that it does not modify the position in parameter space of the response of the open-loop experiment of interest. This non-invasiveness requirement defines a zero-problem whose solutions can be found and tracked in the experiment using path-following methods. Depending on the dynamic features of interest, this zero-problem is often extended to include additional conditions constraining, for instance, an excitation amplitude or a phase difference.

Effectively and reliably tracking the solutions of such zero-problems during tests is challenging. Most existing path-following methods are ideal only in a numerical context where the solution path is smooth and derivatives can be evaluated to high precision. This is not easily achievable in experiments where solutions and derivative estimates are corrupted by measurement noise. In this paper, I investigate the use of an algorithm combining CBC and Gaussian process regression (GPR) to significantly improve the reliability of the continuation process. This algorithm builds on earlier work ${ }^{[7]}$ and was exploited to trace out limit-point bifurcation curves ${ }^{[8]}$. This paper shows how the same algorithm can also be used to identify backbone and NLFR curves. 


\section{EXPERIMENTAL SET-UP}

The set-up considered for the experimental demonstration of the algorithm is a steel cantilever beam whose free end is attached to two linear springs arranged to give rise to geometric nonlinearity at large vibration amplitudes ${ }^{[8]}$. The length of the beam as well as the pre-tension in the springs were carefully adjusted such that a 3:1 modal interaction between the first two bending modes of structure exist. The structure is excited near the clamp using an electrodynamic shaker. The force applied to the structure is measured using a PCB208C03 force transducer and beam tip vibrations are measured using an Omron ZX2-LD100 displacement laser sensor. All measurements are made at $1 \mathrm{kHz}$ sampling with no filtering. The continuation algorithm run on a laptop computer directly connected to the real-time controller (RTC) box via a USB cable. A third-order feedback control law is used to stabilise the dynamics of the system ${ }^{[8]}$.

\section{CONTINUATION PRINCIPLES}

GPR provides local models that are smooth and cheap to evaluate and thus enables the use of established numerical continuation techniques. The GPR models used here possess two inputs which correspond to two experimental adjustable parameters: the excitation frequency $(\omega)$ and one of the fundamental Fourier coefficient of the control target signal $(A)$. To find NLFR curves, GPR is used to model the force amplitude as a function of both input parameters. Tracking NLFRs corresponds to finding the input parameters $(\omega, A)$ such that the applied force is constant. For backbone curves, GPR models the phase difference between the beam response and the applied force as a function of both input parameters. Tracking the backbone curves correspond to finding the input parameters $(\omega, A)$ such that the phase difference is $\pi / 2$. The constant applied force and the phase quadrature conditions define the zero-problems solved by the continuation algorithm when tracking NLFR and backbone curves, respectively.

The algorithm starts by collection an initial set of data points for different values of the input parameters $\omega$ and $A$. Based on the data points captured, a GPR model is built and a solution of the zero problem is found. New data points are then collected to guarantee that the solution found is not an artifact of the model and does not critically depend on the current data. To decide where to collect data in the input parameter space, the current GPR model is exploited to numerically assess the effect of additional data points on the zero-problem. The data point which is found to have the largest effect on the value of the zero-problem is considered to be the most informative and hence is experimentally collected and then added to the model. The procedure is repeated until the effect of any new data point on the zero-problem is below a user-defined threshold. At this stage, the solution of the continuation problem is said to be robust to new data and the continuation algorithm continues to progress in parameter space. The procedure is repeated for every new solution of the zero problem.

\section{RESULTS}

Data points collected using the online continuation algorithm are all included in one GPR model representing the force amplitude as a function of both input parameters. The NLFR curves obtained from this model are shown in solid black in Figure 1(a). Two values of the force amplitude are considered ( $1 \mathrm{~N}$ and $2 \mathrm{~N})$. A similar model is built with the data collected during the backbone continuation. The backbone curve extracted from this model is shown in Figure 1(b). The collection of data points not only at the dynamical feature of interest but also around it enables us to analyse the sensitivity of this feature to experimentally collected data points and highlight regions of greater uncertainty. To visualize this uncertainty, 300 fold curves were numerically computed for different data sets. Each set is based on all the data points collected using the online algorithm but with $10 \%$ of the points, selected randomly, removed. NLFR (backbone) curves obtained with these smaller data sets are shown in gray in Figure 1. For the NLFR, regions of larger result variability, i.e. where the overall curve appears thicker, are located around the resonance. For the backbone curve, larger result variability appears in the softening region at low amplitude. Outside the region where data is available, NLFR and backbone curves are found to diverge very quickly.

\section{CONCLUSIONS}

In this contribution, I show that the algorithm combining control-based continuation with Gaussian process regression introduced in ${ }^{[8]}$ to track fold bifurcation curves can also be exploited track nonlinear frequency response and backbone curves. The variability of the identified features with respect to collected data points can also be assessed. 


\section{ACKNOWLEDGEMENT}

The author gratefully acknowledges the financial support of the Royal Academy of Engineering (Research Fellowship \#RF1516/15/11).

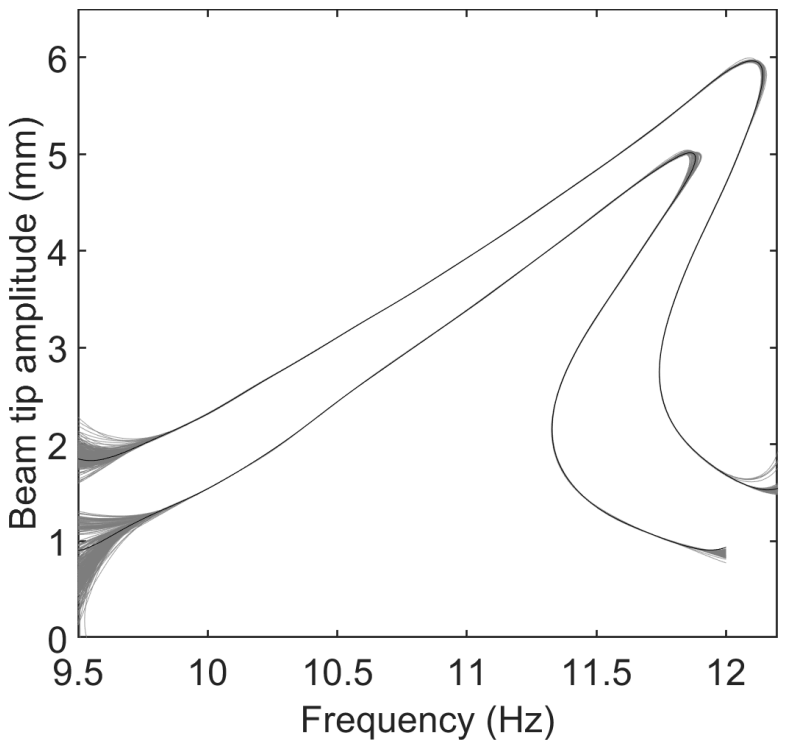

(a)

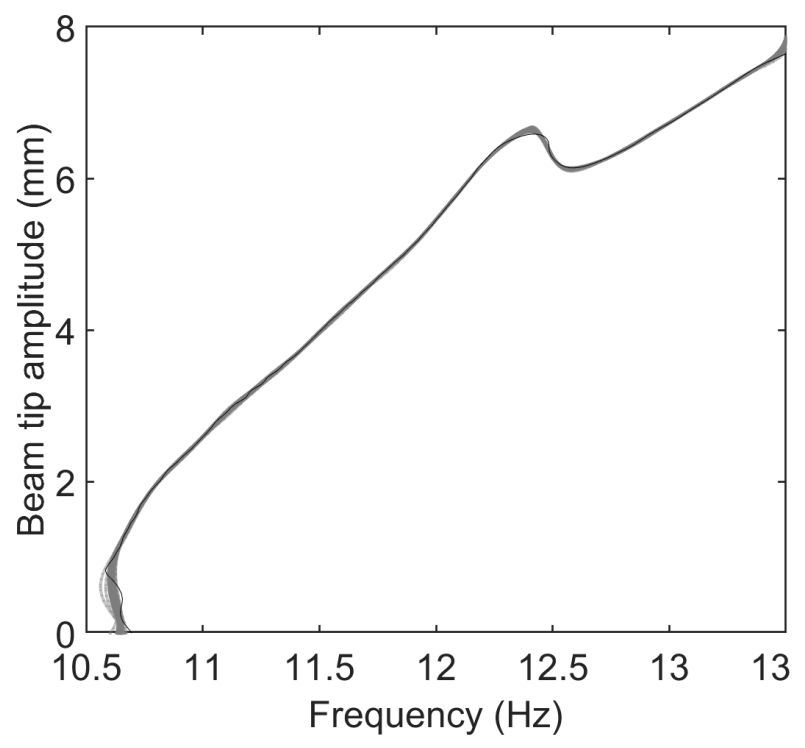

(b)

Figure 1: (a) (-) NLFR curves obtained at $1 \mathrm{~N}$ and $2 \mathrm{~N}$ using all the data points collected. ( - ) NLFR curves obtained with $10 \%$ of the points, selected randomly, removed. (b) (-) Backbone curve obtained using all the data points collected. (-) Backbone curves obtained with $10 \%$ of the points, selected randomly, removed.

\section{References}

[1] D. A. W. Barton and J. Sieber. Systematic experimental exploration of bifurcations with noninvasive control. Physical Review E, 87(5):052916, 2013.

[2] L. Renson, A. Gonzalez-Buelga, D. A. W. Barton, and S. A. Neild. Robust identification of backbone curves using control-based continuation. Journal of Sound and Vibration, 367:145-158, 2016.

[3] L. Renson, A.D. Shaw, D.A.W. Barton, and S.A. Neild. Application of control-based continuation to a nonlinear structure with harmonically coupled modes. Mechanical Systems and Signal Processing, 120:449 - 464, 2019.

[4] J. Sieber and B. Krauskopf. Control based bifurcation analysis for experiments. Nonlinear Dynamics, 51(3):365-377, 2008.

[5] J. Sieber, B. Krauskopf, D. Wagg, A. Gonzalez-Buelga, and S. Neild. Control-based continuation of unstable periodic orbits. Journal of Computational and Nonlinear Dynamics, 6(1):011005, 2010.

[6] E. Bureau, F. Schilder, I. Ferreira Santos, J. J. Thomsen, and J. Starke. Experimental bifurcation analysis of an impact oscillator - tuning a non-invasive control scheme. Journal of Sound and Vibration, 332(22):5883-5897, 2013.

[7] L. Renson, D. A. W. Barton, and S. A. Neild. Experimental tracking of limit-point bifurcations and backbone curves using control-based continuation. International Journal of Bifurcation and Chaos, 27(01):1730002, 2017.

[8] L. Renson, J. Sieber, D. A. W. Barton, A. D. Shaw, and S. A. Neild. Numerical continuation in nonlinear experiments using local gaussian process regression. Nonlinear Dynamics, 2019. 